\title{
Dynamics of quantum discord in the purification process
}

\author{
Wei Song, ${ }^{1}$ Lin Chen, ${ }^{2}$ Ming Yang, ${ }^{3}$ and Zhuo-Liang Cao ${ }^{1}$ \\ ${ }^{1}$ Department of Physics and Electronic Engineering, Hefei Normal University, Hefei 230061, China \\ ${ }^{2}$ Centre for Quantum Technologies, National University of Singapore, 3 Science Drive 2, Singapore 117542 \\ ${ }^{3}$ School of Physics and Material Science, Anhui University, Hefei 230039, China
}

(Dated: October 24, 2018)

\begin{abstract}
We investigate the dynamics of quantum discord during the purification process. In the case of Werner states, it is shown that quantum discord is increased after a round of purification protocol. Furthermore, quantum mutual information and classical correlation is also increased during this process. We also give an analytic expression for a class of higher dimensional states which have additive quantum discord.
\end{abstract}

PACS numbers: 03.67.Mn, 03.65.Ud, 03.65.Ta

It is widedly recognized that entanglment is a key resource and ingredient in the field of quantum information science [1]. However, entanglement is not the only aspect of quantum correlations, and it has been found that there are quantum nonlocality without entanglement [2, 3] and quantum speedup with unentangled states [4 6 ]. Therefore, it is important and interesting to investigate such quantum correlations. In this context, several measures have been proposed to quantify the nonclassical nature of a quantum state such as quantum discord [7, 9], quantum deficit [10], measurement induced disturbance [11]. Furthermore, Modi et al. [12] provides an unified view of quantum and classical correlations recently. Among these measures, quantum discord introduced by Olliver and Zurek [8, 9] is widely used to quantify the quantumness of the correlation contained in a bipartite quantum state. A closely related measure concerning classical correlation was proposed by Henderson and Vedral [7]. Quantum discord is defined as the difference between the total mutual information and the classical correlation contained in a bipartite quantum state. It is more general than entanglement in the sense that separable mixed states can have nonzero quantum discord [13, 14]. It has been suggested that discord is responsible for the speed-up related to deterministic quantum computation with one qubit(DQC1) model [16, 17]. And quantum discord has also attracted much attention for its connection with entanglement irreversibility [18], the entanglement monogamic relation [19], the entanglement in a measurement 20, 21] and the complete positivity [22]. Especially, the operational interpretations of quantum discord through the state merging [23, 24 and locally inaccessible information 25] have established the status of quantum discord as another important resource for quantum informational processing tasks besides entanglement.

On the other hand, quantum discord will degrade due to unavoidable interaction between a quantum system and its environment. It has been shown that quantum discord decays exponentially [26, 27], whereas the entanglement may suffer from sudden death [28] under Markovian noises. The dynamics of quantum discord has also been investigated in a non-Markovian environment [29, 30] and has been demonstrated experimentally un- der both Markovian 31] and non-Markovian [32] environments. In the field of quantum entanglement theory, to achieve a perfect channel in quantum information tasks, entanglement purification is performed to extract a small number of entangled pairs with a relatively high degree of entanglement from a large number of less entangled pairs using only local operations and classical communication (LOCC). Since entanglement is only a special kind of quantum correlations and has different order with other quantum correlations in general. One may ask the question whether quantum correlation can be increased in a round of purification process. In this work we answer this question partly in terms of quantum discord through a concrete example. We also give an analytic expression for a class of higher dimensional states which have additive quantum discord.

In the following discussions we use the quantum discord to measure the quantum correlation. As an example, we shall investigate the dynamics of the quantum discord during the original BBPSSW purification protocol 34]. In the case of Werner states, we find that quantum discord can also be increased after performing the original purification protocol. During one round of purification process, the quantum discord decreases at first and then increases when the intermediate state is transformed into the final state under LOCC. Suppose the noisy Werner state pairs $\rho^{(0)}$ and $\rho^{(1)}$ are given as two copies in the following form:

$$
\begin{aligned}
& \rho^{(0)}=\rho^{(1)}=\rho=F\left|\beta_{11}\right\rangle\left\langle\beta_{11}\right| \\
& +\frac{1-F}{3}\left(\left|\beta_{01}\right\rangle\left\langle\beta_{01}|+| \beta_{10}\right\rangle\left\langle\beta_{10}|+| \beta_{00}\right\rangle\left\langle\beta_{00}\right|\right)(1)
\end{aligned}
$$

where the four Bell states $\beta_{a b} \equiv$ $\frac{1}{\sqrt{2}}\left(|0, b\rangle+(-1)^{a}|1,1 \oplus b\rangle\right)$. We begin with a brief outline of the basic process of BBPSSW purification protocol 34]. Suppose Alice and Bob share two identical noisy Werner state pairs $\rho^{(0)}$ and $\rho^{(1)}$. Firstly, one of them perform $\sigma_{y}$ operations on her/his particles and transform the Werner state pairs into the form: $F\left|\beta_{00}\right\rangle\left\langle\beta_{00}\right|+\frac{1-F}{3}\left(\left|\beta_{10}\right\rangle\left\langle\beta_{10}|+| \beta_{01}\right\rangle\left\langle\beta_{01}|+| \beta_{11}\right\rangle\left\langle\beta_{11}\right|\right)$. Next they apply a bilateral C-Not gate for $\rho^{(0)}$ and $\rho^{(1)}$ 
as the control and target qubits, respectively. Then they bilaterally measure $\rho^{(1)}$ in the computational basis $\{|0\rangle,|| 1\rangle\rangle\}$ and communicate the measurement outcomes to each other. They keep $\rho^{(0)}$ if the measurement outcomes coincide. Otherwise, they discard $\rho^{(0)}$. After performing the measurement, the original mixed Werner state $\rho^{(0)}$ will be mapped into a new mixed state $\rho^{\prime}$ described as:

$$
\begin{aligned}
\rho^{\prime} & =\frac{10 F^{2}-2 F+1}{8 F^{2}-4 F+5}\left|\beta_{00}\right\rangle\left\langle\beta_{00}\left|+\frac{6 F-6 F^{2}}{8 F^{2}-4 F+5}\right| \beta_{10}\right\rangle\left\langle\beta_{10}\right| \\
& +\frac{2 F^{2}-4 F+2}{8 F^{2}-4 F+5}\left|\beta_{01}\right\rangle\left\langle\beta_{01}\left|+\frac{2 F^{2}-4 F+2}{8 F^{2}-4 F+5}\right| \beta_{11}\right\rangle\left\langle\beta_{11}\right|
\end{aligned}
$$

In fact, the mixed state $\rho^{\prime}$ is only an intermediate state and hence it cannot be the initial state in the second round of BBPSSW purification. We perform $\sigma_{y}$ operations firstly and then twirl the state applying at random one of the four operations $\left\{\sigma_{x} \otimes \sigma_{x}, \sigma_{y} \otimes \sigma_{y}, \sigma_{z} \otimes \sigma_{z}, I \otimes I\right\} \quad 35$ locally to each party of the pair. In this way we transform the mixed state $\rho^{\prime}$ into the Werner state: $\chi=F^{\prime}\left|\beta_{11}\right\rangle\left\langle\beta_{11}\right|+$ $\frac{1-F^{\prime}}{3}\left|\beta_{01}\right\rangle\left\langle\beta_{01}\left|+\frac{1-F^{\prime}}{3}\right| \beta_{10}\right\rangle\left\langle\beta_{10}\left|+\frac{1-F^{\prime}}{3}\right| \beta_{00}\right\rangle\left\langle\beta_{00}\right|$, with $F^{\prime}=\frac{10 F^{2}-2 F+1}{8 F^{2}-4 F+5}$. The mixed state $\chi$ is the input state in the next round of BBPSSW purification. Because $F^{\prime}>F$ in the range of $\frac{1}{2}<F<1$, using the iteration of the protocol we can probabilistic extract a mixed state pair with a relatively high degree of entanglement from two entangled copies using only LOCC. In order to compare the quantum correlation during the purification process, we compute the quantum discord of the initial mixed state $\rho$, the intermediate state $\rho^{\prime}$ and the final state $\chi$, respectively.

Firstly, we recall the definitions of quantum discord. To define quantum discord, we starts with the quantum mutual information defined as $\mathcal{I}\left(\rho_{A B}\right)=S\left(\rho_{A}\right)+$ $S\left(\rho_{B}\right)-S\left(\rho_{A B}\right)$, where $S(\rho)=-\operatorname{Tr}\left(\rho \log _{2} \rho\right)$ is the von Neumann entropy. The quantum mutual information is regarded as quantifying the total correlation in the mixed state $\rho_{A B}$, then the quantum discord is defined as

$$
\mathcal{D}\left(\rho_{A B}\right)=\mathcal{I}\left(\rho_{A B}\right)-\mathcal{C}\left(\rho_{A B}\right)
$$

where $\mathcal{C}\left(\rho_{A B}\right)$ denote the classical correlation of the state $\rho_{A B}$ and it is defined as $\mathcal{C}\left(\rho_{A B}\right)=$ $\max _{\left\{\Pi_{A}\right\}}\left[S\left(\rho_{A}\right)-S\left(\rho_{A B} \mid\left\{\Pi_{k}\right\}\right)\right]$, where the maximum is $\left\{\Pi_{k}\right\}$

taken over the set of projective measurements $\left\{\Pi_{k}\right\}$ and $S\left(\rho_{A B} \mid\left\{\Pi_{k}\right\}\right)=\sum_{k} p_{k} S\left(\rho_{k}\right)$ is the conditional entropy of system A, with $\rho_{k}=\frac{\operatorname{Tr}_{B}\left(\Pi_{k} \rho_{A B} \prod_{k}\right)}{p_{k}}$ and $p_{k}=\operatorname{Tr}_{A B}\left(\rho_{A B} \prod_{k}\right)$. Here, we only consider projective measures because Hamieh et al. [36] have shown that for a two-qubit system the projective measurement is the positive operator-valued measure (POVM) which maximizes classical correlation. Consider the Bell-diagonal states of the form [37]

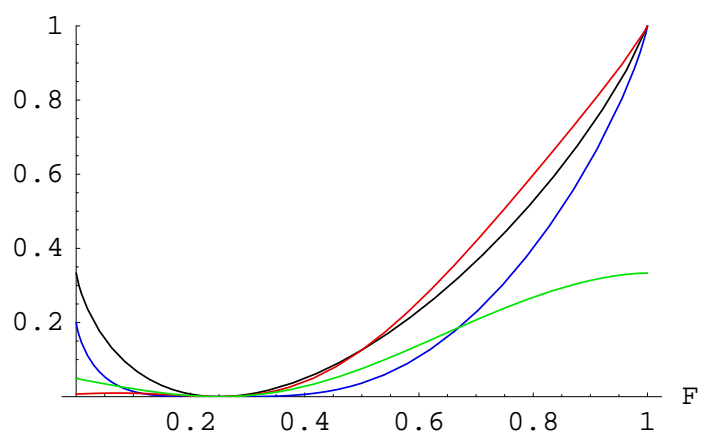

FIG. 1: (Color online). The black, blue, red and green curves represent the quantum discord as a function of $F$ correspond to $\mathcal{D}(\rho), \mathcal{D}\left(\rho^{\prime}\right), \mathcal{D}(\chi)$ and $\mathcal{D}\left(\chi^{\prime}\right)$ respectively. Here $\rho$ denotes the initial state, $\rho^{\prime}$ denotes the intermediate state, $\chi$ denotes the final state, and $\chi^{\prime}$ denotes the intermediate state after performing a random bilateral $\mathrm{SU}(2)$ rotation locally on each qubits.

$$
\rho_{A B}=\frac{1}{4}\left(I+\sum_{j=1}^{3} c_{j} \sigma_{j}^{A} \otimes \sigma_{j}^{B}\right)=\sum_{a, b} \lambda_{a b}\left|\beta_{a b}\right\rangle\left\langle\beta_{a b}\right|
$$

with eigenvalues

$$
\lambda_{a b}=\frac{1}{4}\left(1+(-1)^{a} c_{1}-(-1)^{a+b} c_{2}+(-1)^{b} c_{3}\right)
$$

An analytical formula of quantum discord have been obtained for Bell-diagonal states and can be expressed as [38]:

$$
\begin{aligned}
Q & =\frac{1}{4}\left[\left(1-c_{1}-c_{2}-c_{3}\right) \log _{2}\left(1-c_{1}-c_{2}-c_{3}\right)\right. \\
& +\left(1-c_{1}+c_{2}+c_{3}\right) \log _{2}\left(1-c_{1}+c_{2}+c_{3}\right) \\
& +\left(1+c_{1}-c_{2}+c_{3}\right) \log _{2}\left(1+c_{1}-c_{2}+c_{3}\right) \\
& \left.+\left(1+c_{1}+c_{2}-c_{3}\right) \log _{2}\left(1+c_{1}+c_{2}-c_{3}\right)\right] \\
& -\frac{1-c}{2} \log _{2}(1-c)-\frac{1+c}{2} \log _{2}(1+c)
\end{aligned}
$$

where $c \equiv \max \left\{\left|c_{1}\right|,\left|c_{2}\right|,\left|c_{3}\right|\right\}$. For the mixed state of Eq.(2), we have

$$
c_{1}=\frac{16 F^{2}-8 F+1}{8 F^{2}-4 F+5}, c_{2}=-c_{1}, c_{3}=\frac{12 F-3}{8 F^{2}-4 F+5}
$$

It is directly to see that $c_{1}$ is always a nonnegative value, and the maximum value among them is $\left|c_{3}\right|$. Thus, we can calculate the quantum discord of $\rho^{\prime}$ according to the formula in Eq. (6). In Fig. 1 we plot the discord $\mathcal{D}\left(\rho^{\prime}\right)$ as a function of $F$. In order to make a comparison with the original discord before the purification process, we also plot $\mathcal{D}(\rho)$ in Fig. 1. We can see that the quantum 


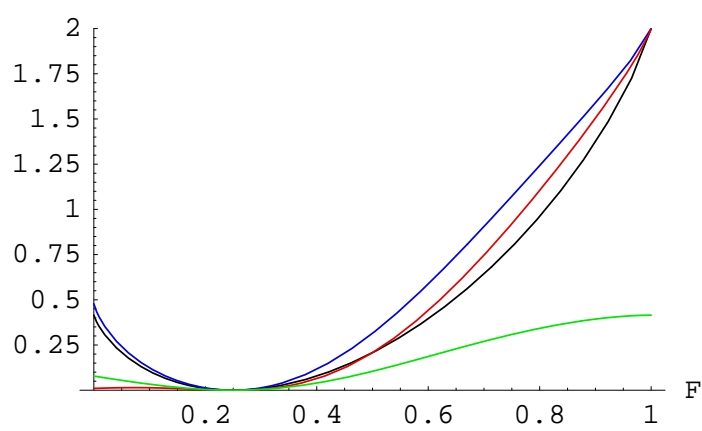

FIG. 2: (Color online). Dynamics of quantum mutual information $\mathcal{I}(\rho)$ (black line), $\mathcal{I}\left(\rho^{\prime}\right)$ (blue line), $\mathcal{I}(\chi)$ (red line) and $\mathcal{I}\left(\chi^{\prime}\right)$ (green line) respectively.

discord of the intermediate state always decreases for arbitrary $F$ compared to the initial state. Using the same method, we can also compute $\mathcal{D}(\chi)$ and plot it in the same figure. It is shown that quantum discord of the final state is increased after one round of purification process, which indicates that the purification protocol can also purify the quantum discord at the same time. To summarize the above discussions, the quantum discord of the original Werner states experiences two phases during one round of purification protocol. During the first process the quantum discord decreases when the initial Werner states are transformed into the intermediate state. During the second process quantum discord increases when the intermediate state is transformed into the final state under LOCC. For the special case $F=\frac{1}{4}$, the initial Werner state has a zero discord. In this case, it has the form $\rho=\sum_{i} p_{i}|i\rangle\langle i| \otimes \rho_{i}$ where $|i\rangle$ is the orthonormal bases. We can increase its discord to a nonzero value by transforming this classical state into the nonclassical form under LOCC. Thus, we conclude that LOCC can be used to increase discord of a bipartite quantum state both for the zero and non-zero cases.

To further understanding of the evolution of quantum discord under LOCC. Suppose we perform a random bilateral $\mathrm{SU}(2)$ rotation locally on each side of $\rho^{\prime}$ directly and transform it into the following Werner state: $\chi^{\prime}=F^{\prime \prime}\left|\beta_{11}\right\rangle\left\langle\beta_{11}\left|+\frac{1-F^{\prime \prime}}{3}\right| \beta_{01}\right\rangle\left\langle\beta_{01}\left|+\frac{1-F^{\prime \prime}}{3}\right| \beta_{10}\right\rangle\left\langle\beta_{10}\right|+$ $\frac{1-F^{\prime \prime}}{3}\left|\beta_{00}\right\rangle\left\langle\beta_{00}\right|$, with $F^{\prime \prime}=\frac{2 F^{2}-4 F+2}{8 F^{2}-4 F+5}$. We also plot the quantum discord of $\chi^{\prime}$ in Fig. 1. We can see that the quantum discord is decreased in this case. It indicates that we can decrease the quantum discord by LOCC. In Fig. 2 and Fig. 3 we also plot the dynamics of quantum mutual information and classical correlation during the purification process, respectively. We find that they are both increased after a round of purification protocol for $\frac{1}{2}<F<1$. Under the LOCC operation from the intermediate state $\rho^{\prime}$ to the final state $\chi$ or $\chi^{\prime}$, we find that the evolution of quantum mutual information and classical correlation is always nonincreasing.

Before ending this paper, we want to provide a class of higher-dimensional mixed states with computable quan-

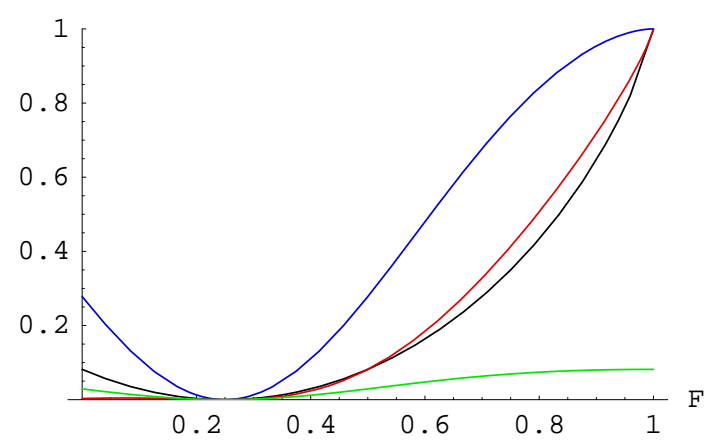

FIG. 3: (Color online). Dynamics of classical correlation $\mathcal{C}(\rho)$ (black line), $\mathcal{C}\left(\rho^{\prime}\right)$ (blue line), $\mathcal{C}(\chi)$ (red line) and $\mathcal{C}\left(\chi^{\prime}\right)$ (green line) respectively.

tum discord of the form: $\rho_{A B}=\sum_{i j} p_{i j}\left|a_{i}\right\rangle|i\rangle\left\langle a_{j}\right|\langle j|$, where $\left|a_{i}\right\rangle$ is not restricted to the orthonormal bases. For these class of state, we have $C\left(\rho_{A B}\right)=S\left(\rho_{A}\right)$, because the minmum is obtained by using the projector $|i\rangle\langle i|$ to get $\left|a_{i}\right\rangle\left\langle a_{i}\right|$ as the resulting state. Thus we have $\mathcal{D}\left(\rho_{A B}\right)=S\left(\rho_{B}\right)-S\left(\rho_{A B}\right)$. For the general case, the classical correlations are super-additive [7] and thus the quantum discord is subadditive. However, for the special mixed state $\rho_{A B}$ mentioned above, we can prove its quantum discord is additive. Recalling the Koashi-Winter monogamy relation [39] for quantum correlations within a pure tripartite state $|\psi\rangle_{A B C}: E_{F}\left(\rho_{A C}\right)+C\left(\rho_{A B}\right)=S_{A}$ . Notice the definition of discord, it is further shown that the above relation can be rewritten as [40]

$$
E_{F}\left(\rho_{A C}\right)=\mathcal{D}\left(\rho_{A B}\right)+S(A \mid B),
$$

where $S(A \mid B)=S(A B)-S(B)$ denotes the quantum conditional entropy of $\rho_{A B}$ and $E_{F}$ denote the entanglement of formation. We can further define its regularized version [41] entanglement cost $E_{C}(\rho)=\lim _{n \rightarrow \infty} \frac{1}{n} E_{F}\left(\rho^{\otimes n}\right)$ and $\mathcal{J}\left(\rho_{A B}\right)=\lim _{n \rightarrow \infty} \frac{1}{n} \mathcal{D}\left(\rho^{\otimes n}\right)$, then we have:

$$
E_{C}\left(\rho_{A C}\right)=\mathcal{J}\left(\rho_{A B}\right)+S(A \mid B)
$$

It is shown in Ref.[18] that the equality above gives a operational meaning to the regularized version of quantum discord as a measure of the amount of entanglement loss when Alice and Bob distill entanglement by hashing. If we choose $\rho_{A B}$ as the above mixed states, then we can write its purified form as: $|\psi\rangle_{A B C}=\sum_{i} \lambda_{i}\left|a_{i}\right\rangle|i\rangle\left|b_{i}\right\rangle$, where $\left|b_{i}\right\rangle$ may not be orthogonal for different $i$. It is directly to see that $\rho_{A C}$ is a separable state with zero entanglement formation and entanglement cost. Thus by Eq.(8) and Eq.(9) we have that quantum discord of the special mixed state $\rho_{A B}$ mentioned above is additive.

In the above discussions we have investigated the dynamics of quantum discord during the purification process. It is found that quantum discord is increased after a 
round of purification protocol. We show that during the original BBPSSW purification protocol quantum discord can also be increased at the same time. Therefore, we conclude that quantum discord of arbitrary two-qubit state can be increased by the original purification protocol. However, for the higher-dimensional case, we do not know whether quantum discord is also increased. At present, we cannot answer this question due to the lack of analytical formula of quantum discord for the higherdimensional case.

Wei Song was supported by National Natural Science Foundation of China under Grant No.10905024, the Key
Project of Chinese Ministry of Education under Grant No.211080, the Doctoral Startup Foundation of Hefei Normal University under Grant No.2011rcjj03 and the Key Program of the Education Department of Anhui Province under Grant No. KJ2011A243. The Centre for Quantum Technologies is funded by the Singapore Ministry of Education and the National Research Foundation as part of the Research Centres of Excellence program. Ming Yang was supported by the Key Project of Chinese Ministry of Education.(No.210092). Zhuo-Liang Cao was supported by the National Natural Science Foundation of China under Grant No.11005029.
[1] M. A. Neilsen and I. L. Chuang, Quantum Computation and Quantum Information (Cambridge University Press, New York, 2000).

[2] C. H. Bennett, D. P. DiVincenzo, C. A. Fuchs, T. Mor, E. Rains, P. W. Shor, J. A. Smolin, and W. K. Wootters, Phys. Rev. A 59, 1070 (1999).

[3] M. Horodecki, P. Horodecki, R. Horodecki, J. Oppenheim, A. Sen, U. Sen, and B. Synak-Radtke, Phys. Rev. A 71, 062307 (2005).

[4] S. L. Braunstein, C. M. Caves, R. Jozsa, N. Linden, S. Popescu, and R. Schack, Phys. Rev. Lett. 83, 1054 (1999).

[5] D. A. Meyer, Phys. Rev. Lett. 85, 2014 (2000).

[6] A. Datta, S. T. Flammia, and C. M. Caves, Phys. Rev. A 72, 042316 (2005); A. Datta and G. Vidal, ibid. 75, 042310 (2007).

[7] L. Henderson and V. Vedral, J. Phys. A 34, 6899 (2001).

[8] W. H. Zurek, Annalen der Physik, 9, 855 (2000).

[9] H. Ollivier and W. H. Zurek, Phys. Rev. Lett. 88, 017901 (2001).

[10] A. K. Rajagopal and R. W. Rendell, Phys. Rev. A 66, 022104 (2002).

[11] S. Luo, Phys. Rev. A 77, 022301 (2008).

[12] K. Modi, T. Paterek, W. Son, V. Vedral, and M. Williamson, Phys. Rev. Lett. 104, 080501 (2010).

[13] B. Dakic, V. Vedral, and C. Brukner, Phys. Rev. Lett. 105, 190502 (2010).

[14] L. Chen, E. Chitambar, K. Modi, and G. Vacanti, Phys. Rev. A 83, 020101 (R) (2011).

[15] A. Ferraro, L. Aolita, D. Cavalcanti, F. M. Cucchietti, A. Acin, Phys. Rev. A 81, 052318 (2010).

[16] E. Knill and R. Laflamme, Phys. Rev. Lett. 81, 5672 (1998).

[17] A. Datta, A. Shaji, and C. M. Caves, Phys. Rev. Lett. 100, 050502 (2008).

[18] M. F. Cornelio, M. C. de Oliveira and F. F. Fanchini, Phys. Rev. Lett. 107, 020502 (2011).

[19] F. F. Fanchini, M. C. de Oliveira, L. K. Castelano, M. F. Cornelio, arXiv:1110.1054 (2011).

[20] M. Piani, S. Gharibian, G. Adesso, J. Calsamiglia, P. Horodecki and A. Winter, Phys. Rev. Lett. 106, 220403 (2011).

[21] A. Streltsov, H. Kampermann and D. Bruss, Phys. Rev.
Lett. 106, 160401 (2011).

[22] A. Shabani and D. A. Lidar, Phys. Rev. Lett. 102, 100402 (2009).

[23] D. Cavalcanti, L. Aolita, S. Boixo, K. Modi, M. Piani and A. Winter, Phys. Rev. A 83, 032324 (2011).

[24] V. Madhok and A. Datta, Phys. Rev. A 83, 032323 (2011).

[25] F. F. Fanchini, L. K. Castelano, M. F. Cornelio, M. C. de Oliveira, New Journal of Physics 14, 013027 (2012).

[26] T. Werlang, S. Souza, F. F. Fanchini, and C. J. Villas Boas, Phys. Rev. A 80, 024103 (2009).

[27] J. Maziero, L. C. Celeri, R. M. Serra, and V. Vedral, Phys. Rev. A 80, 044102 (2009).

[28] T. Yu and J. H. Eberly, Science. 323, 598 (2009).

[29] B. Wang, Z.-Y. Xu, Z.-Q. Chen, and M. Feng, Phys. Rev. A 81, 014101 (2010).

[30] F. F. Fanchini, T. Werlang, C. A. Brasil, L. G. E. Arruda, A. O. Caldeira, Phys. Rev. A 81, 052107 (2010).

[31] Jin-Shi Xu, Xiao-Ye Xu, Chuan-Feng Li, Cheng-Jie Zhang, Xu-Bo Zou, and Guang-Can Guo, Nat. Commun. 1,7 (2010).

[32] Jin-Shi Xu, Chuan-Feng Li, Cheng-Jie Zhang, Xiao-Ye $\mathrm{Xu}$, Yong-Sheng Zhang, and Guang-Can Guo, Phys. Rev. A 82,042328 (2010).

[33] M. Horodecki, P. Horodecki, and R. Horodecki, Phys. Rev. Lett. 80, 5239 (1998).

[34] C. H. Bennett, G. Brassard, S. Popescu, B. Schumacher, J. A. Smoin, and W. K. Wootters, Phys. Rev. Lett. 76, 722 (1996).

[35] R. F. Werner, Phys. Rev. A 40, 4277 (1989).

[36] S. Hamieh, R. Kobes, and H. Zaraket, Phys. Rev. A 70, 052325 (2004).

[37] M. D. Lang, and C. M. Caves, Phys. Rev. Lett. 105, 150501 (2010).

[38] S. Luo, Phys. Rev. A 77, 042303 (2008).

[39] M. Koashi and A. Winter, Phys. Rev. A 69, 022309 (2004).

[40] F. F. Fanchini, M. F. Cornelio, M. C. de Oliveira, A. O. Caldeira, Phys. Rev. A 84, 012313 (2011).

[41] I. Devetak and A. Winter, IEEE Tran. Info. Theory. 50, 3183 (2004). 